\title{
Der Wert Alpiner Forschungseinzugsgebiete im Bereich der Fernerkundung, der Schneedeckenmodellierung und der lokalen Klimamodellierung
}

\author{
Matthias Bernhardt · Stefan Härer · Moritz Feigl · Karsten Schulz
}

Online publiziert: 25. Juli 2018

(C) Der/die Autor(en) 2018

Zusammenfassung Der Hochgebirgsraum ist traditionell ein Raum mit wenigen bis keinen Messstationen. Vorortmessungen sind nur in ausgesuchten Gebieten oder nur punktuell verfügbar. Aus diesem Grund werden Landoberflächenmodelle, regionale Klimamodelle und Fernerkundungsdaten eingesetzt, um die benötigte Information zur erzeugen. Wie diese Daten im Gebirgsraum tatsächlich sind, bleibt dabei oftmals offen, da Evaluationsdatensätze fehlen, oder nur lückenhaft vorhanden sind. Besonders wertvoll sind in diesem Zusammenhang alpine Einzugsgebiete, die - meist aufgrund der Bemühungen von regionalen Gruppen oder Organisationen - einen höheren Grad an Instrumentierung aufweisen. Das vom bayerischen Umweltministerium geförderte Einzugsgebiet um die Umweltforschungsstation Schneefernerhaus an der Zugspitze (D) ist ein solches Gebiet. Die drei diskutierten Fallbeispiele zeigen, mit welchen Unsicherheiten zu rechnen ist, wenn Modelle gänzlich ohne oder mit wenig Basisdaten aus dem Zielgebiet betrieben werden. Weiterhin soll der Nutzen alpiner Forschungseinzugsgebiete im Kontext optimierter Modellsetups, verbesserter Modellergebnisse und abgeleiteter Fernerkundungsprodukte aufgezeigt werden.

Assoc. Prof. Dr. M. Bernhardt ( $₫)$. Dipl.-Geogr. S. Härer · DI M. Feigl · Univ.-Prof. Dipl.-Geoökol. Dr. K. Schulz Institut für Wasserwirtschaft, Hydrologie und konstruktiven Wasserbau (IWHW), Universität für Bodenkultur Wien, Muthgasse 18, 1190 Wien, Österreich matthias.bernhardt@boku.ac.at
The importance of Alpine research catchments for model evaluation and for the improvement of remote sensing products

Abstract Mountain areas are traditionally data sparse since meteorological or gauging stations are rare or nonexistent. Field measurements are only available for some areas, or for a few measuring points, within the catchment. Hence, data from land surface models, climate models and remote sensing have become increasingly important sources of alternative data. However, the quality of these data sources is often undefined given the limitations of available actual observations. Data from Alpine research catchments often exist only due to the efforts of local research groups in the region, providing extremely valuable data that are based on a high level of instrumentation. The research catchment Zugspitze (RCZ) located in the direct environment of the environmental research station Schneefernerhaus is such a local effort, which is mainly funded by the Bavarian ministry for the Environment. In three case studies, we will demonstrate the importance of such data and show how strongly uncertainties from modelling and remote sensing can be reduced.

\section{Einleitung}

Die europäischen Alpen sind ein extrem wichtiger geografischer Raum, wenn es um die Wasserversorgung des Gebirgslands und die der Vorländer geht (Viviroli et al. 2011). Wasserversorgung kann in diesem Zusammenhang sehr umfassend verstanden werden. In erster Linie steigern die Alpen aufgrund ihres sehr günstigen Abflusskoeffizienten das generelle Wasserdargebot in den Gerinnen (Mankin et al. 2015; Wada et al. 2011; Viviroli et al. 2007). Was- ser aus alpinen Einzugsgebieten wird für die allgemeine Wasserversorgung, als Brauchwasser, für die Bewässerung, als Energieträger, als Freizeitfaktor in Skigebieten etc. intensiv genutzt. Eine Veränderung des Wasserdargebots im Raum oder über die Zeit hätte damit eklatante und weitreichende Folgen. Im Rahmen des globalen Klimawandels sind aber gerade die Gebirgsregionen vulnerabel (Pomeroy et al. 2015), das Klimawandelsignal ist hier deutlich stärker als im angrenzenden Flachland (Weber et al. 2016). Aus diesem Umstand lassen sich zukünftig deutliche Veränderungen im alpinen Wasserkreislauf ableiten. Die Qualität von Zukunftsprognosen, die die Ausprägung dieser Veränderung abzubilden vermögen, und die Güte der Beschreibung des momentanen Wasserkreislaufs hängen dabei stark von den verfügbaren Beobachtungsdaten ab. Klemes (1990) beschrieb den Gebirgsraum noch als eines der schwärzesten Löcher im Bereich der Hydrologie und beschrieb die Schwierigkeiten bei der Datenerfassung mit dem Bild, dass ein Team von olympischen Skifahrern vonnöten wäre, um valide Daten in dem vorliegenden rauen Terrain und unter den gegebenen klimatischen Bedingungen zu erheben. Seit dieser Zeit haben sich Modelle, seien es Landoberflächen- oder Klimamodelle stark entwickelt und auch die Fernerkundung ist $\mathrm{zu}$ einem wichtigen Datenlieferanten geworden (Bernhardt 2008; Bernhardt and Schulz 2010; Gao et al. 2012, 2017; Harer et al. 2013; Gutman et al. 2016). Allerdings benötigen Modelle sowie Fernerkundungsprodukte zumindest einige gut instrumentierte Gebiete, in denen Daten zur Modellentwicklung, zur Validierung und zur Kalibrierung erhoben werden können (Pomeroy et al. 2015). Das International Network for Alpine Research Catchment Hydrology (INARCH) (Bernhardt et al. 2015) hat es sich zur Aufgabe gemacht, gut instrumentierte alpine 


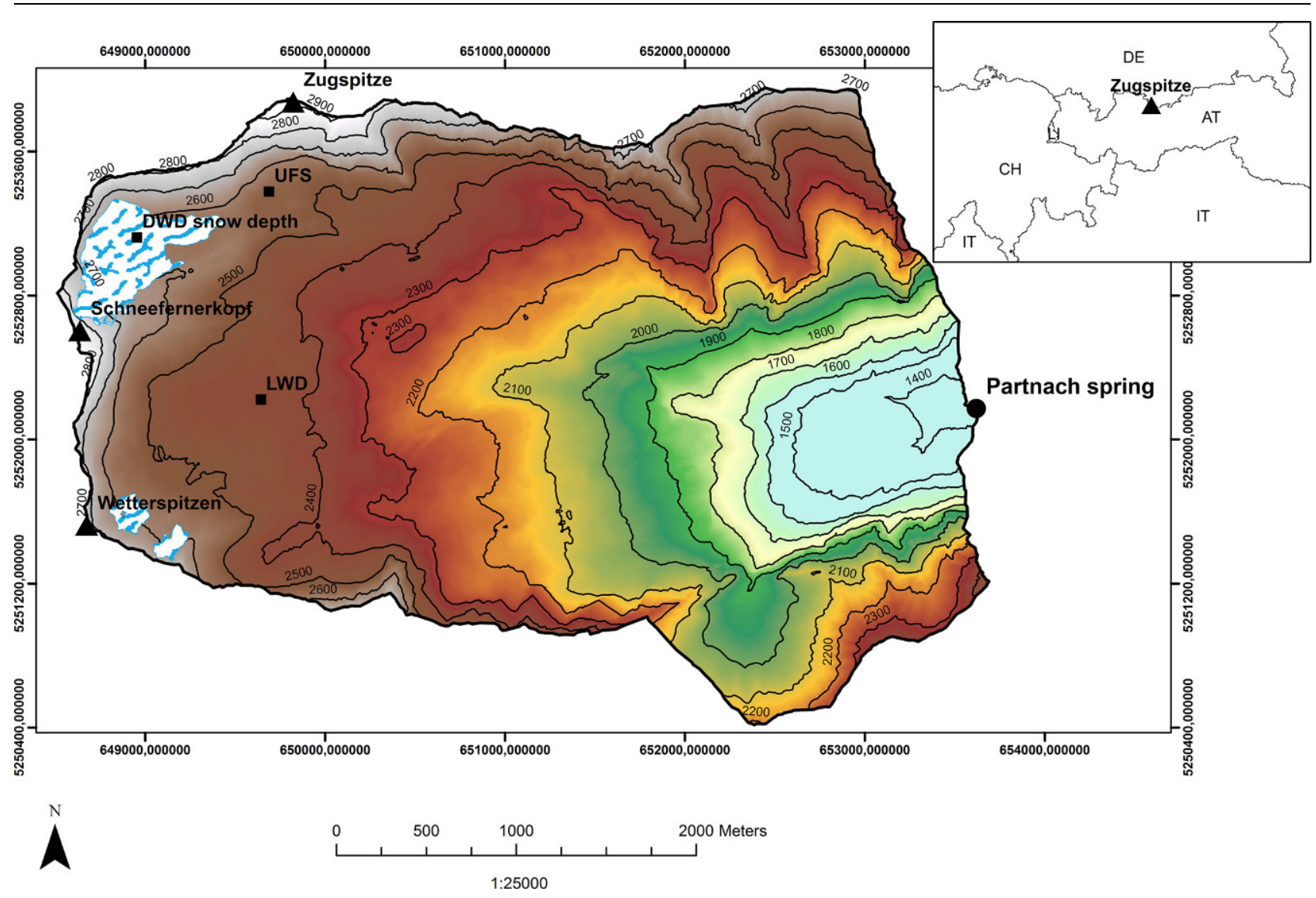

Abb. 1 Das Research Catchment Zugspitze (RCZ). Eingetragen sind die DWD-Station an der Zugspitze (Zugspitze) und die Station des Lawinenwarndienstes $(L W D)$, die im Wesentlichen die Daten für diese Publikation liefern

Einzugsgebiete $\mathrm{zu}$ vernetzen und die erhobenen Daten der allgemeinen Wissenschaftsgemeinschaft zur Verfügung zu stellen. In diesem Artikel werden Daten aus einem INARCH-Einzugsgebiet, dem Research Catchment Zugspitze (RCZ) (Bernhardt et al. 2014), genutzt, um zum einen aufzuzeigen, inwieweit komplexere prozessbasierte Schneedeckenmodelle auf eine Veränderung von Gebietscharakteristiken reagieren, inwieweit Daten aus hoch gelegenen Einzugsgebieten wichtig sind, um zu einem adäquaten Setup von regionalen Klimamodellen kommen zu können und schlussendlich darzustellen, wie Fernerkundungsprodukte aufgrund von lokalen Messungen verbessert werden können.

Fallbeispiel I geht der Frage nach, inwieweit Klimawandelprognosen auf benachbarte, aber topografisch unterschiedliche Einzugsgebiete übertragbar sind. Der Fokus liegt hierbei auf der Schneedeckenmodellierung. Im Experiment wird das prozessbasierte Landoberflächenmodell CRHM (Lopez-Moreno et al. 2016; Pomeroy et al. 2007) verwendet, um zu einer Beschreibung der Schneedeckenentwicklung im Gebiet zu gelangen. Das Versuchslayout sieht vor, dass das Testgebiet jeweils in 90 Grad-Schritten um die zentral gelegene meteorologische Station des Lawinenwarndienstes gedreht wird. Die Eingangsdaten bleiben dabei unverändert. Durch die neue Orientierung verändern sich die Einstrahlungsgeometrien im Gelände. Diese Veränderung wird im Modell berücksichtigt und zeigt Effekte v.a. in der Ablationsperiode. Im Falle eines kalibrierten Tag-Grad-Modells wäre dies nicht der Fall. Dieses würde bei konstanten Eingangsdaten ein unverändertes Verhalten zeigen. Das Fallbeispiel soll aufzeigen, dass komplexe prozessbasierte Modelle sich aufgrund ihrer Formulierung an neue Gebiete adaptieren. Dies ist v. a. bei der Frage eines Transfers von Modellen in nichtinstrumentierte Gebiete von Bedeutung.

Fallbeispiel II: Regionale Klimamodelle gewinnen als Datenlieferanten für Landoberflächenmodelle zunehmend an Gewicht (Gutmann et al. 2016). Das regionale Klimamodell WRF (Gutmann et al. 2012) nimmt dabei eine Ausnahmestellung ein, da es regelmäßig als Treiberdaten-Generator für Studien weltweit eingesetzt wird (Givati et al. 2012; Warrach-Sagi et al. 2013). WRF ist dabei eines der weitestverbreiteten Modelle, hat aber den Nachteil, dass es eine enorme Computerleistung benötigt, wenn es $\mathrm{zu}$ hochaufgelösten Produkten kommt (Gutmann et al. 2016). Dies führt zu der Tatsache, dass für die meisten Studien nur ein Standardsetup zum Einsatz kommt, d.h. die zahlreich vorhandenen Modellparameter nicht angepasst werden, da sich Sensitivitätsstudien aufgrund der Rechenzeit verbieten. Im vorgelegten Fallbeispiel wird das Intermediate Complex Atmospheric Model (ICAR) (Gutmann et al. 2016), das aus der gleichen Entwicklergruppe stammt wie WRF, verwendet, um aufzuzeigen, inwiefern Daten aus alpinen Einzugsgebieten nützlich sein können, um regionale Simulationen zu verbessern.

Fallbeispiel III: Fernerkundungsprodukte spielen in der Erforschung des 

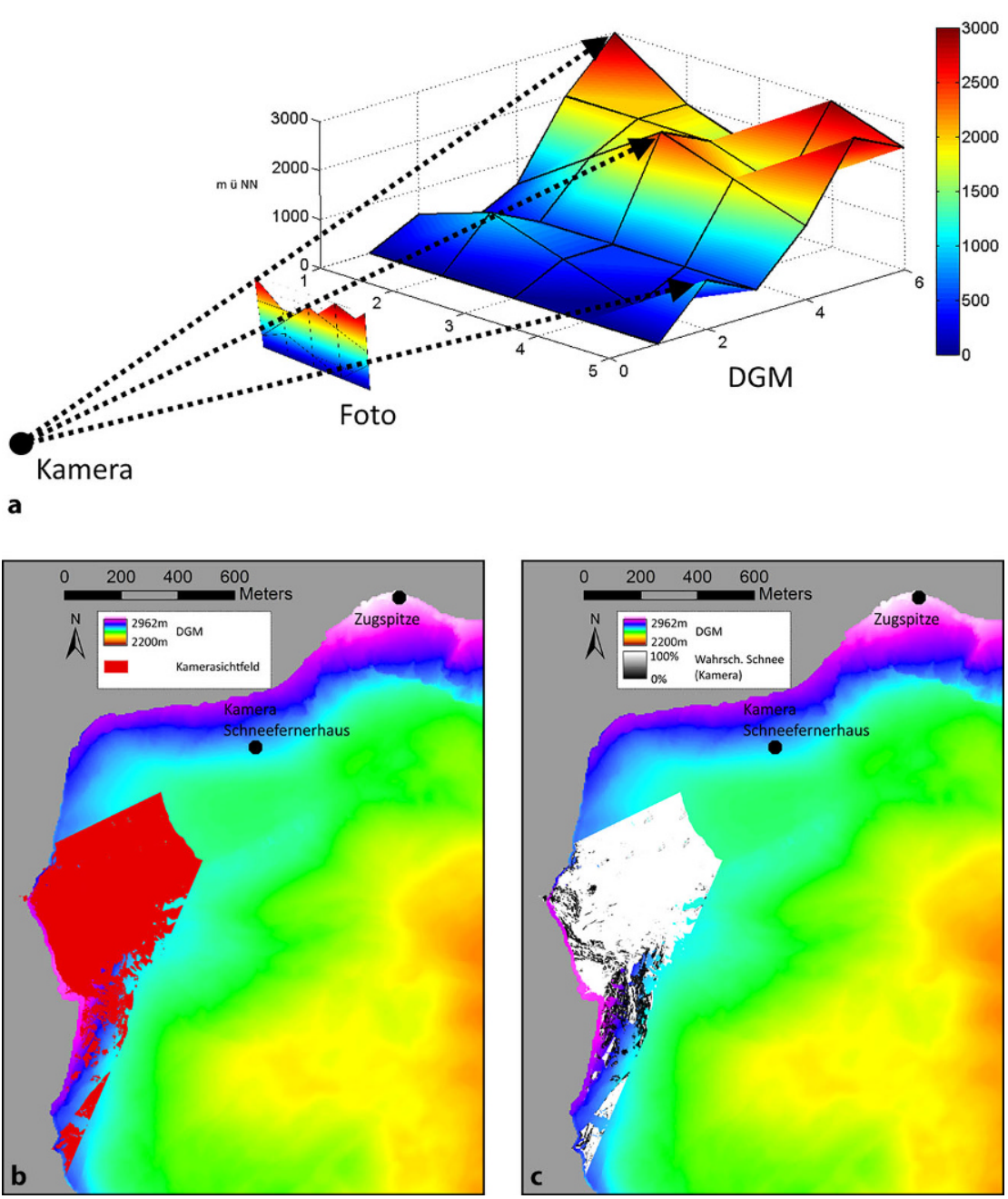

Abb. 2 SchematischeDarstellung derFunktionsweise von PRACTISE(a) und eine Darstellung der Ergebnisfelder (b, c)

alpinen Raums eine wichtige Rolle, da sie zuverlässig und kontinuierlich Daten über einen schwer zu erfassenden topografischen Raum liefern. Dabei werden die mehrkanaligen Informationen der Satellitenbilder oftmals in thematische Informationen überführt. Im alpinen Bereich wird z. B. sehr oft die schneebedeckte Fläche aus Satellitendaten extrahiert (Arsenault et al. 2014; Ault et al. 2006; Bitner et al. 2002). Essenziell ist dabei in den meisten Fällen der Normalized Differenced Snow Index (NDSI) (Dozier 1989). Dieser Ansatz erlaubt auf einfache Weise die Generierung von Schneedeckenkarten. Wird der NDSI-Ansatz auf ein Satellitenbild angewendet, erhält man statt Reflektanzen NDSI-Werte zwischen -1 und 1 . Um zu einer Ableitung der Schneedecke $\mathrm{zu}$ kommen benötigt es zudem einen $\mathrm{zu}$ definierenden Grenzwert, der fest-

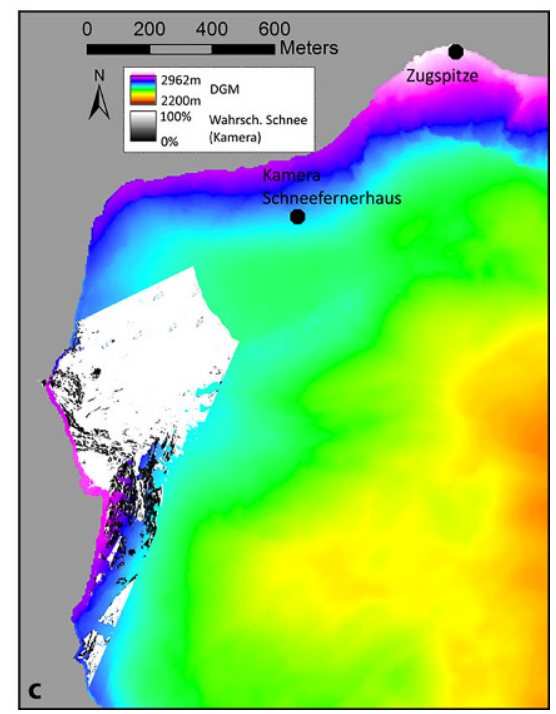

den höchsten Punkt des $11,4 \mathrm{~km}^{2}$ großen Einzugsgebiets (Abb. 1) dar. Bei einer Jahresdurchschnittstemperatur (1981-2010) von $-4,5^{\circ} \mathrm{C}$ fallen auf der Zugspitze im Mittel $2080 \mathrm{~mm}$ Niederschlag pro Jahr. Das EZG ist oberhalb von $2000 \mathrm{~m}$ ü. NN, bis auf Pionierpflanzen und alpine Rasengesellschaften, frei von Vegetation. Aufgrund der Karstformen und der glazialen Überprägung weist die Oberfläche des Zugspitzplatts eine große Heterogenität auf. Die besondere geologische Situation führt dazu, dass das EZG allein über den Partnachursprung entwässert wird und somit als natürlicher Lysimeter angesehen werden kann. Dies und die hervorragende Ausstattung des EZG mit insgesamt sechs automatischen Wetterstationen sowie die gute Zugänglichkeit machen es zu einem idealen „Freilandlabor" für hydrologische Studien. Weitere Details zum Untersuchungsgebiet finden sich in Weber et al. (2016) und Rappl et al. (2010).

\section{Methoden}

Um die Lesbarkeit zu erleichtern, werden die Methoden der drei Fallbeispiele in drei separaten Unterkapiteln dargestellt.

\subsection{Fallbeispiel I}

Die Modellierung der Schneedeckenentwicklung im EZG erfolgt mit dem prozessbasierten Cold Regions Hydrological Model (CRHM) (Pomeroy et al. 2007). Aufgrund der Instrumentierung mit Strahlungssensoren im Einzugsgebiet wird es möglich, ein prozessbasiertes Schneedeckenmodell zu betreiben. Dieses nutzt neben der Lufttemperatur auch die kurzwellige und die langwellige Einstrahlung zur Beschreibung des Ablationsprozesses der Schneedecke (Marks et al. 1999).

$$
Q_{m}+Q_{n}+Q_{H}+Q_{E}+Q_{G}+Q_{D}=\frac{d U}{d t}
$$
einzugsgebiete geeignet sind, einen kalibrierten, intersubjektiven und zuverlässigen Grenzwert zu ermitteln.

\section{Gebietsbeschreibung}

Das Research Catchment Zugspitze (RCZ) (Abb. 1) wird durch den Quellaustritt der Partnach am Partnachursprung $(1430 \mathrm{~m}$ ü. NN) definiert. Es befindet sich in den Nördlichen Kalkalpen und ist Teil des Zugspitzgebiets. Mit $2962 \mathrm{~m}$ ü. NN stellt die Zugspitze
Wobei $\mathrm{Q}_{\mathrm{m}}$ die verfügbare Energie für die Schneeschmelze darstellt, $\mathrm{Q}_{\mathrm{n}}$ die Nettostrahlung, $\mathrm{Q}_{\mathrm{H}}$ den turbulenten Wärmefluss der fühlbaren Energie, $Q_{E}$ den turbulenten Wärmefluss der latenten Energie, $Q_{G}$ den Bodenwärmestrom und $\mathrm{Q}_{\mathrm{D}}$ Wärmestrom aus externen Quellen, wie z.B. Regen. 


\begin{tabular}{|c|c|c|c|}
\hline & Eingangsdaten & Prozessierung & Ausgangsdaten \\
\hline 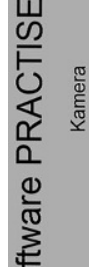 & $\begin{array}{l}\text { - DGM } \\
\text { - Foto } \\
\text { - Externe und interne } \\
\text { Kameraparameter } \\
\text { - Passpunkte für } \\
\text { Optimierung }\end{array}$ & $\begin{array}{l}\text { 1. Optimierung } \\
\text { Kameraparameter } \\
\text { 2. Analyse } \\
\text { Kamerasichtfeld } \\
\text { 3. Projektion Foto in } \\
\text { DGM } \\
\text { 4. Schneeklassifikation }\end{array}$ & $\begin{array}{l}\text { - Kamerasichtfeld } \\
\text { - Fotoschneekarte }\end{array}$ \\
\hline 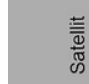 & $\begin{array}{l}\text { - Satellitenbild } \\
\text { - Wolkenmaske }\end{array}$ & $\begin{array}{l}\text { 1. Maskierung Wolken } \\
\text { 2. NDSI Berechnung }\end{array}$ & $\begin{array}{l}\text { - NDSI Satellitenkarte } \\
\text { - Satellitenschneekarte }\end{array}$ \\
\hline
\end{tabular}

Abb. 3 Ablaufschema der verschiedenen Projektions- und Klassifikationsphasen in PRACTISE
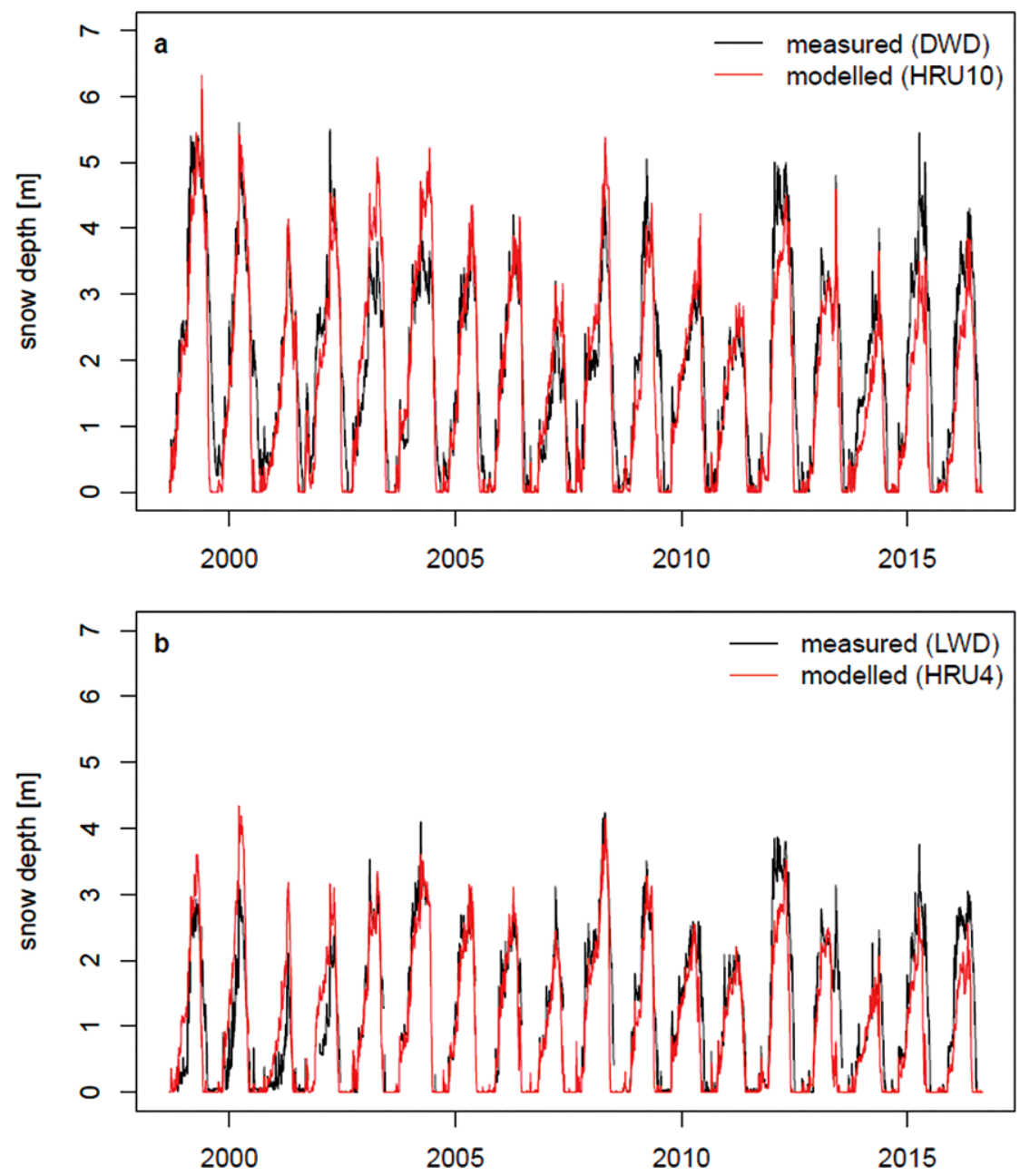

Abb. 4 Übereinstimmung zwischen modellierterund gemessenerSchneehöhean den Stationen des Deutschen Wetterdienstes und des Bayerischen Lawinenwarndienstes (Abb. 1)
Die Schneeschmelze wird dann über folgende Formulierung ermittelt:

$$
M=\frac{Q_{m}}{\rho_{w} B h_{f}}
$$

Hier steht $\rho_{w}$ für die Dichte des Wassers, B ist der Anteil von Eis an einer Einheit feuchten Schnees und $h_{f}$ ist die latente Schmelzenergie von Eis. Die benötigten Strahlungsterme werden auf Basis der Messungen an die Geländegeometrie angepasst. Die Anpassung der direkten und der diffusen Strahlung an das Gelände erfolgt über Garnier and Ohmura (1970), die Anpassung der Schneealbedo über Gray and Landine (1987) die der langwelligen Strahlung nach Sicart et al. (2004), die Modifizierung der Strahlung beim Durchtritt durch Vegetation nach Pomeroy and Dion (1996) und die Nettostrahlung wird nach Granger and Gray (1990) adaptiert. Unter Berücksichtigung dieser Formelsätze soll im Rahmen der vorgestellten Studie untersucht werden, wie sich eine Veränderung der Exposition des Gebiets auf die Schneedeckenentwicklung auswirkt. Die klimatologischen Treiberwerte werden konstant gehalten, das RCZ aber in drei Schritten um jeweils $90^{\circ}$ gedreht. Ein Temperaturindexmodell (Hock 2003), wie es in vielen operationellen Anwendungen verwendet wird, reagiert naturgemäß nicht auf eine solche Modifikation, da die Treiberdaten konstant bleiben. Das physikalisch basierte Modell hingegen modifiziert die Strahlungsterme nach den genannten Formelsätzen gemäß der Exposition und Hangneigung und erzeugt in der Folge expositionsabhängige Unterschiede.

In CRHM wird das EZG mithilfe von Hydrological Response Units (HRUs) (Flügel 1995; Zehe et al. 2014) räumlich diskretisiert. HRUs sind dabei Raumeinheiten, von denen angenommen wird, dass sie sich aufgrund ihrer naturräumlichen Ausstattung hydrologisch gleich bzw. vergleichbar verhalten. Die Einteilung der HRUs erfolgte auf Basis eines Clusteransatzes, der verschiedene Parameter wie Höhe, Neigung, Orientierung, Kurvatur und den Wind Shelter Index (Erickson et al. 2005) berücksichtigt. Die erzeugten Cluster wurden dann auf Basis von terrestrischen LIDAR-Aufnahmen evaluiert, indem überprüft wurde, ob die clusterbasierte HRU-Verteilung mit den allgemeinen Charakteristika der Schneeverteilung übereinstimmt. Nachdem dies gewährleistet werden konnte, wurden 
Tab. 1 Modellierte und gemessene maximale SWE an der Zugspitze

\begin{tabular}{llllllllll} 
& Zugspitze & Zugspitze & Zugspitze-90 & Zugspitze-90 & Zugspitze-180 & Zugspitze-180 & Zugspitze-270 & Zugspitze-270 \\
& $1980-2015$ & $2070-2100$ & $1980-2015$ & $2070-2100$ & $1980-2015$ & $2070-2100$ & $1980-2015$ & $2070-2100$ \\
\hline Winter & 740,29 & 203,10 & 744,88 & 211,23 & 734,40 & 205,27 & 734,60 & 204,55 \\
\hline Spring & 927,28 & 240,07 & 933,53 & 250,56 & 918,98 & 241,51 & 918,38 & 240,47 \\
\hline Summer & 293,62 & 7,93 & 291,05 & 7,73 & 268,15 & 7,62 & 268,56 & 7,64 \\
\hline Autumn & 292,63 & 75,65 & 294,77 & 78,38 & 288,98 & 76,08 & 288,66 & 75,74
\end{tabular}

Modellläufe mithilfe von historischen Daten 1980-2015 und Prognoseläufe von 2070-2100 durchgeführt. Die historischen Daten wurden dabei genutzt, um eine Vergleichsgrundlage zu bilden und um die Modellgüte anhand von vorhandenen Schneehöhenmessungen zu überprüfen. Die Prognoseläufe wurden mithilfe von WETTREG-Datensätzen durchgeführt (Kreienkamp et al. 2013), die eine statistisch generierte zukünftige Meteorologie für die DWDStation an der Zugspitze bereitstellen.

\subsection{Fallbeispiel II}

Für die regionale Klimamodellierung kommt das Modell ICAR des National Center for Atmospheric Research (NCAR) (Gutmann et al. 2016) zum Einsatz. ICAR ist ein simplifiziertes dreidimensionales Atmosphärenmodell. Im vorliegenden Fall werden Wärme- und Feuchteflüsse über ein, mit der linearen Mountainwave Theorie (Barstad und Gronas 2006; Smith 1979) modifiziertes, Zirkulationsfeld bestimmt, das aus dem zu skalierenden Modell ERA Interim (Dee et al. 2011) übernommen wird. Die Übernahme der Zirkulationsdynamik aus dem zu skalierenden grobskaligen Atmosphärenmodell stellt dabei den wesentlichen Unterschied zu komplexeren regionalen Klimamodellen (RCMs) wie WRF dar. Dieser Ansatz vermeidet, dass in ICAR direkt die Navier-Stokes-Gleichung für Bewegung gelöst werden muss, die traditionell den Kern von RCMs darstellt. Zur Konservierung der Masse und zum Ausgleich lokaler räumlicher Ungleichgewichte im Windfeld wird aber zusätzlich die vertikale Luftbewegung berechnet (Gutmann et al. 2016). Dies geschieht v. a., um bei konvergenten Windströmen numerische Probleme zu vermeiden. Die sonstigen Formulierungen in ICAR ähneln bzw. sind ident mit denen in komplexeren RCMs. Im Bereich der Mikrophysik stehen ein simplifiziertes Schema nach Smith und Barstad (2004), das etablierte Thompson-Schema (Thompson et al.
2008) sowie das komplexere MorrisonSchema (Morrison et al. 2005b) zur Verfügung. Diese drei Schemata werden in der vorgelegten Studie verwendet und die resultierenden Unterschiede im RCZ aufgezeigt. Weiterhin werden Parameter wie die Cloud Droplet Concentration im Morrison-Schema und die Schneefallgeschwindigkeit verändert. Die Cloud Droplet Concentration kann nach Morrison (2005a) Werte zwischen 100 bis 300 Wolkentröpfchen pro $\mathrm{cm}^{3}$ annehmen. In unseren Fall wurde der Standard von 300 Wolkentröpfchen pro $\mathrm{cm}^{3}$ einem modifizierten Wert von 150 gegenübergesetzt, der für maritim beeinflusste Regionen vorgesehen ist. Die Schneefallgeschwindigkeit wurde mit dem Standardwert (Morrison et al. 2005a) und einem modifizierten Parametersatz nach Lin et al. (1983) berechnet. Als zu skalierendes Modell nutzen wir ERA-Interim für den Zeitraum 01.09.2012-01.05.2013. Dabei wird auf den Cycle 31r2 des ECMWF's Integrated Forecast Systems (IFS) für das ERAInterim-Produkt zurückgegriffen (Dee et al. 2011). ERA-Interim verfügt dabei über 60 vertikale Modellschichten, wobei die oberste Domain durch das 0,1-hpa-Druckniveau definiert ist. Die räumliche Darstellung erfolgt über ein reduziertes Gauß'sches Grid (N128) und erreicht eine nahezu uniforme Auflösung von $79 \mathrm{~km}$. Über ICAR wird diese Auflösung für den weiteren Bereich um das RCZ auf $1 \mathrm{~km}$ skaliert.

Eine detaillierte Übersicht aller Modellroutinen und der genutzten Parameter findet sich in Gutmann et al. (2016).

\subsection{Fallbeispiel III}

Für die Kalibrierung der NDSI-basierten Schneedeckenkarten wurde auf die an der BOKU am Institut für Hydrologie und Wasserwirtschaft (HyWa) entwickelte Software PRACTISE (Harer et al. 2013; Härer et al. 2016) zurückgegriffen. PRACTISE wird verwendet, um zweidimensionale Fotografien in das Gelände projizieren zu können (Abb. 2). Die pro- jizierten Daten werden in einem zweiten Schritt klassifiziert, mit dem Ziel eine hochauflösende Schneedeckenkarte zu generieren (Abb. 2). Die der Projektion zugrunde liegende Technik geht auf einen Ansatz aus der Computeranimation nach Watt und Watt (1992) zurück.

Für die Projektion der Fotografie müssen der Kamerastandort und 13 Kameraparameter bekannt, sowie der Bildmittelpunkt geografisch verortet werden. Da die benötigten Ortsangaben und Kameraparameter in der Realität häufig nicht exakt bestimmt werden können bzw. von den Herstellerangaben abweichen und auch schon minimale Abweichungen zu deutlichen Fehlern im Endprodukt führen können, werden die Parameter über einen dynamical dimension search (DDS)Algorithmus (Tolson and Shoemaker 2007) optimiert. Wenn der optimale Parametersatz gefunden wurde, wird das Sichtfeld der Kamera nach einem Ansatz von Wang et al. (2000) berechnet. Die Projektion findet dann nach dem Ansatz von Watt und Watt (1992) statt und das projizierte Bild wird im Folgenden mit einer Methodik nach Salvatori et al. (2011) klassifiziert. Als Endprodukt erhält man ein Bild, das entweder der Auflösung des im Rahmen der Prozessierung verwendeten DGMs oder aber der Auflösung der Kamera entspricht. Dies hängt davon ab, welcher der beiden Faktoren der limitierende ist.

Im Satellitenbild wird aus den infraroten Kanälen der Normalised Difference Snow Index (NDSI) berechnet. Die Kalibrierung des NDSI-Grenzwertes geschieht über eine schrittweise Modifikation des Grenzwertes zwischen -1 und 1. Schlussendlich wird der Grenzwert selektiert, der die größte Redundanz zwischen der Schneedeckenkarte aus dem Satellitenbild und der lokal abgeleiteten Schneedeckenkarte aus PRACTISE erzeugt. Das generelle Ablaufschema in PRACRTISE ist in Abb. 3 dargestellt. 


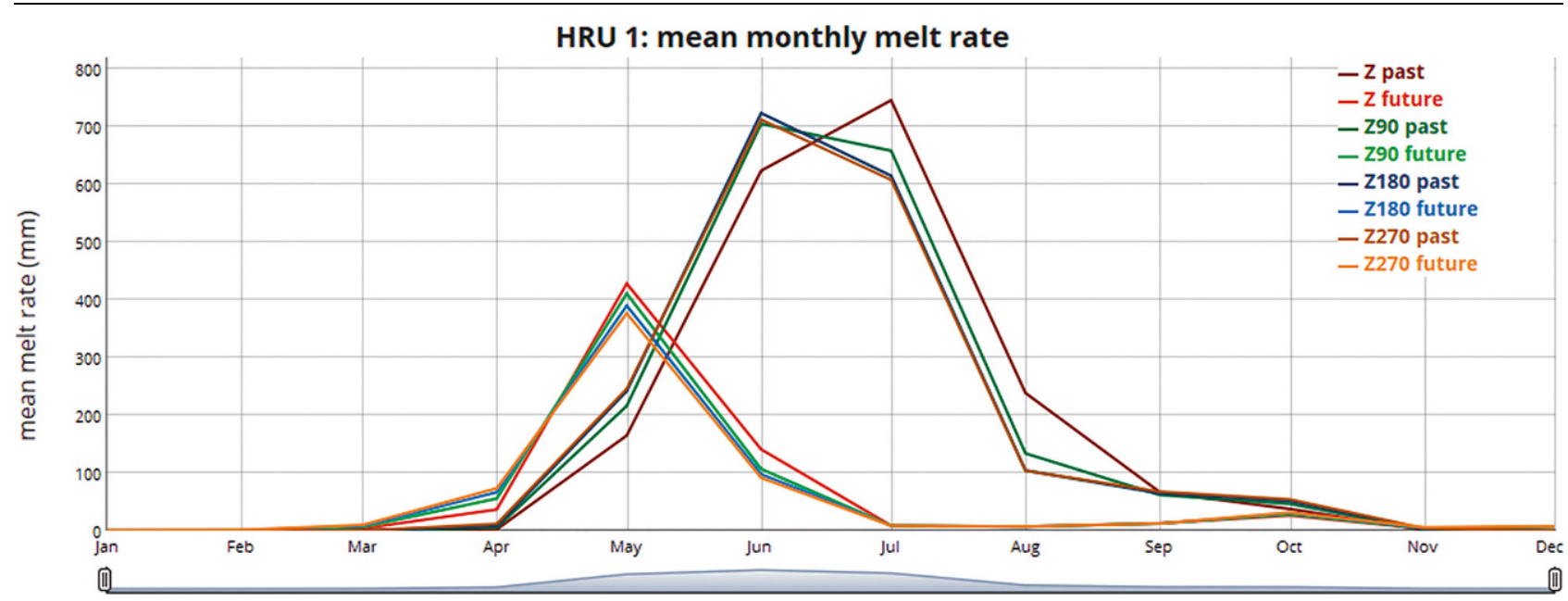

Abb. 5 Historische und zukünftige Verteilung der monatlichen Schmelzraten in Abhängigkeit von der Orientierung des Gebiets

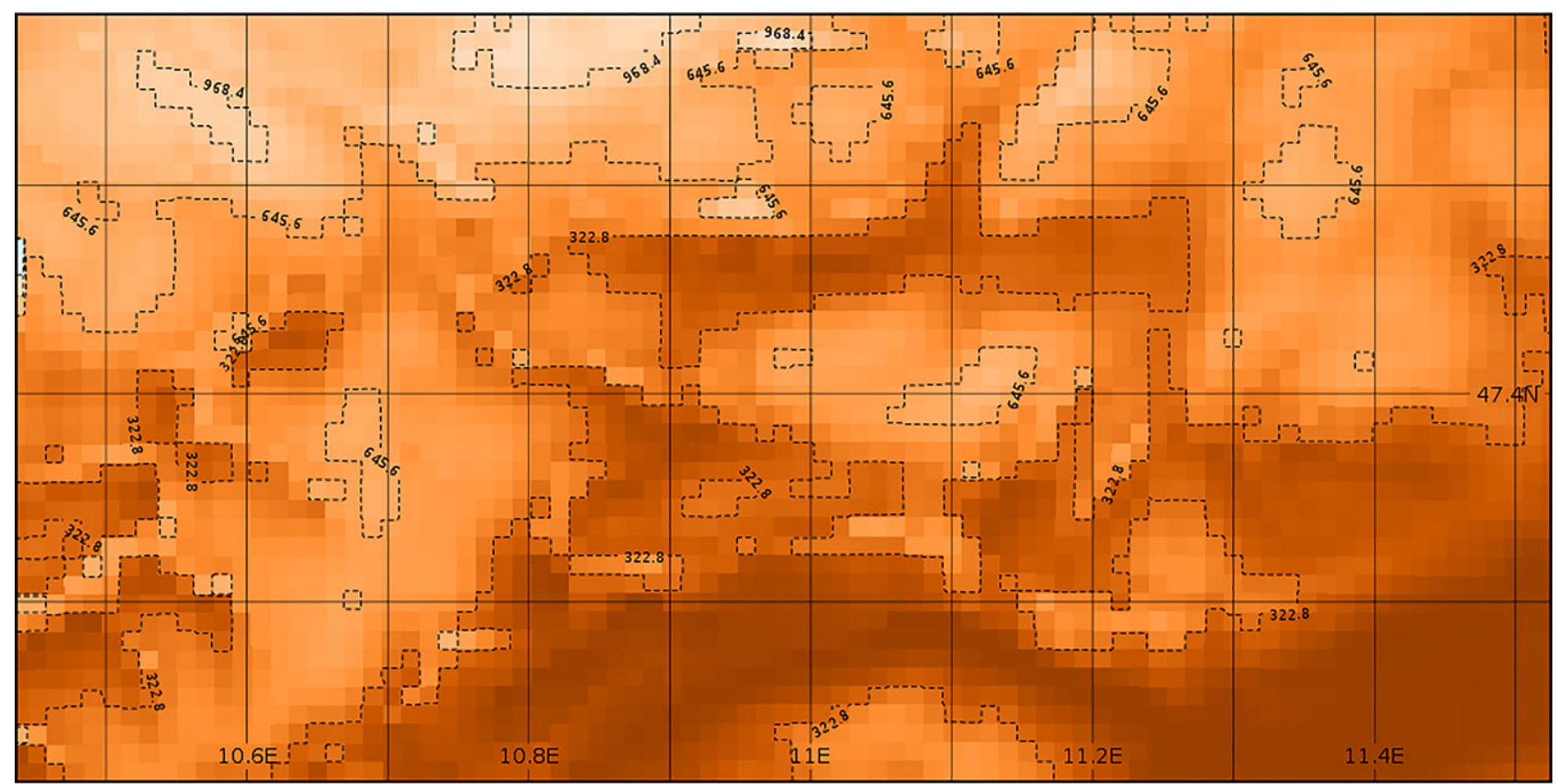

Combined large scale and convective rain, snow and graupel (accumulated) ( $\mathrm{kg} \mathrm{m}-2$ )

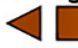

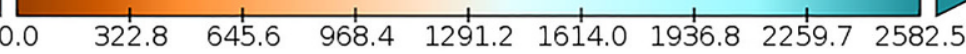

Data $\operatorname{Min}=0.0 . \operatorname{Max}=1570.4$

Abb. 6 Niederschlagsfeld auf Basis des Mikrophysik-Schemas von Smith und Barstad (2004)

\section{Ergebnisse und Diskussion}

\subsection{Fallbeispiel I}

In einem ersten Schritt wurde die Modelgüte des Schneedeckenmoduls an zwei Standorten im Gebiet, für den Zeitraum 1998-2016 überprüft. Die Schneehöhenmessungen und die Modellergebnisse zeigen hier eine sehr gute Übereinstimmung. Über die Drehung des Gebietes um jeweils 90 Grad wird nun der hypothetische Einfluss einer anderen Topografie auf das Ergebnis untersucht. Es werden somit 3 synthetische Einzugsgebiete erzeugt (Abb. 4).

Nachdem die Modellgüte zumindest für diesen Modellversuch als ausreichend $\mathrm{zu}$ bewerten ist, wird nun untersucht, inwieweit sich die Drehung des Gebietes auf die Entwicklung des Schneewasseräquivalentes (SWE) im Gebiet und auf die Schneeschmelze im
Gebiet ausprägt. Weiterhin wird untersucht, ob die Orientierung einen Einfluss auf die Reaktion des Gebietes auf das Klimawandelsignal hat. Für diese Untersuchung werden WETTREGDaten mit einem Temperaturtrend von $4 \mathrm{~K}$ belegt. Im Generellen ist $\mathrm{zu}$ sagen, dass die WETTREG-Szenarien, wenn man die Zeiträume 1961-2010 und 2050-2099 zugrunde legt, eine deutliche Verminderung des Niederschlags vorhersagen $(-32$ bis $-38 \%$, je 
Tab. 2 Gemessene und modellierte Niederschlagshöhen für die DWD-Stationen Garmisch-Partenkirchen und Zugspitze

\begin{tabular}{|c|c|c|c|c|}
\hline Schema & $\begin{array}{l}\text { Garmisch gemessen } \\
(\mathrm{mm})\end{array}$ & $\begin{array}{l}\text { Garmisch modelliert } \\
(\mathrm{mm})\end{array}$ & $\begin{array}{l}\text { Zugspitze gemessen } \\
(\mathrm{mm})\end{array}$ & $\begin{array}{l}\text { Zugspitze modelliert } \\
(\mathrm{mm})\end{array}$ \\
\hline Smith \& Barstad (2004) & 627 & 268 & 1173 & 662 \\
\hline Thompson et al. (2008) & - & 889 & - & 1644 \\
\hline Morrison et al. (2005a) & - & 628 & - & 1511 \\
\hline Morrison mod. Fallgeschwindigkeit & - & 693 & - & 1666 \\
\hline $\begin{array}{l}\text { Morrison mod. Cloud Droplet Concen- } \\
\text { tration }\end{array}$ & - & 623 & - & 1528 \\
\hline
\end{tabular}

nach Realisation). Der sommerliche Rückgang im Niederschlag von $-20 \%$ in den Alpen korrespondiert mit dem mittleren sommerlichen Rückgang des Niederschlags für ganz Deutschland (Spekat et al. 2007) und entspricht in der Tendenz den bekannten ECHAM5-Regionalisierungen (Kling et al. 2012 und Smiatek et al. 2009). Allerdings zeigen im Rahmen von WETTREG auch die Winter in den Alpen einen drastischen Niederschlagsrückgang von $15 \%$. Dies und die gestiegenen Temperaturen erklären den starken Rückgang im winterlichen SWE an der Zugspitze (vgl. Abb. 3).

Vergleicht man die historischen und die zukünftigen maximalen SWE an der Zugspitze (Tab. 1), so wird deutlich, dass die Orientierung des Gebietes wenig Einfluss auf die Modellergebnisse hat. Dies kann dadurch erklärt werden, dass während des Schneedeckenaufbaus im Beobachtungsgebiet die Strahlung eine untergeordnete Rolle im Rahmen der Schneedeckendynamik spielt. Diese Aussage gilt in ihrer Tendenz nur für die diskutierten Höhenlagen und ist in Tallagen mit häufigen Schmelzereignissen, auch in der Akkumulationsperiode, so nicht haltbar. Für das RCZ ist das Bild jedoch eindeutig. Im Rahmen von Langfristprognosen und für die Berechnung des maximalen SWE hat die Orientierung des Gebiets nahezu keine Auswirkung auf das Ergebnis (Tab. 1).

Weiterhin überwiegt der Trend aus den Klimadaten in seinem Effekt den erzielten Effekt aus der Drehung deutlich.

Anders stellt sich die Situation dar, wenn die Schneeschmelze im Jahresgang betrachtet wird (Abb. 5), hier lassen sich durchaus Unterschiede feststellen. So zeigt sich das Maximum in der Schneeschmelze in Abhängigkeit von der Orientierung um bis zu einen Monat verschoben. Dies lässt sich darauf zurückführen, dass durch die Drehung die Exposition der vorhandenen HRUs zur Sonne verändert wird. Wird diese in Summe größer, wird auch die empfangene Energiemenge und damit die Schmelze nach Gl. 2 ebenfalls höher. Dies ist ein relevanter Aspekt, wenn die Schneedecke mit kalibrierten Modellen aus Nachbareinzugsgebieten oder entfernteren Einzugsgebieten betrieben werden sollen, die jedoch die veränderten Geländeeigenschaften bei der Modellierung nicht berücksichtigen. Hier entstehen in der Folge schnell Fehleinschätzungen in Bezug auf den Zeitpunkt der maximalen Schmelze, die wie im gezeigten Beispiel in der Größenordnung von einem Monat liegen können. Es empfiehlt sich hier bei der Prognose oder im Management, Modelle zu selektieren, die die Schmelze nicht nur über die Temperatur approximieren. Diese interpolieren die Temperatur meist nur höhenabhängig und wären daher für die gezeigten Realisationen zum selben Ergebnis gekommen, da sich am Zusammenhang zwischen Temperatur und Höhe in keinem Beispiel etwas verändert.

Modelle hingegen, die die Schneeschmelze über die Energiebilanz berechnen, reagieren auf Veränderungen im Terrain, insofern adäquate Formelsätze genutzt werden, und sind daher für eine Modellierung, die zumindest ein Mindestmaß an einer lokalen Ausprägung der Schneedecke interessiert sind, besser geeignet. Interessant ist auch, dass sich der Unterschied in den Modellergebnissen für den Zeitraum 2070-2100 nivelliert. Dies lässt sich aber anhand der WETTREG-Daten erklären, die für die Zukunftsszenarios ab Mai in allen Orientierungen ein Übermaß an Schmelzenergie bereitstellen und somit die Geländeeigenschaften nahezu belanglos werden. Auch dies ist ein interessantes Ergebnis, da es zumindest darauf hindeutet, dass Gebiete, die bisher aufgrund ihrer Sonderlage ein besonders stabiles Schneeregime aufweisen, diesen Vorzug in Zukunft verlieren könnten, da das vorhandene Energiedargebot die schützenden Wirkungen der Sonderlage mehr als aufbraucht.

\subsection{Fallbeispiel II}

In der Fallstudie II werden Unterschiede in der prognostizierten Niederschlagsverteilung mit dem Modell ICAR und 3 unterschiedlichen Ansätzen zur Darstellung der Mikropyhsik im Bereich der Wolkendynamik untersucht. Die Ergebnisse sind in den Abb. 6, 7 und 8 für die Modelldomain dargestellt. Das sehr stark vereinfachte Schema nach Smith und Barstad (2004) erzeugt ein Niederschlagsfeld mit sehr kleinen räumlichen Gradienten. Die Werte spannen sich hier zwischen $301 \mathrm{~mm}$ in den Tallagen und $972 \mathrm{~mm}$ in den Gipfellagen auf (Abb. 6). Nimmt man die Stationen in GarmischPartenkirchen $(627 \mathrm{~mm})$ und Zugspitze $(1173 \mathrm{~mm})$ als Referenz, wird deutlich, dass dieses Schema, mit Werten von $268 \mathrm{~mm}$ für Garmisch und $662 \mathrm{~mm}$ für die Zugspitze (Tab. 2), den Niederschlag an beiden Standorten deutlich unterschätzt. Mit dem Thompson-Schema wird für Garmisch ein Niederschlagswert von $886 \mathrm{~mm}$ und für die Zugspitze ein Wert von $1644 \mathrm{~mm}$ erreicht (Abb. 7). Für das Morrison-Schema sind es 628 und $1511 \mathrm{~mm}$ (Abb. 8). Es wird deutlich, dass die Selektion der jeweiligen Mikrophysik zur Beschreibung der Wolkendynamik im Modell erheblichen Einfluss auf die Modellergebnisse hat. Um abschätzen zu können, welche der Schemata im Gebiet tauglich sind, ist eine Ausstattung mit höhergelegenen Stationen unumgänglich. Stehen diese zur Verfügung, wird deutlich, dass die zwei komplexeren Schemata eine deutlich bessere Realisierung der wahren Begebenheiten im Bereich des RCZ ermöglichen. Weitere Stationen, wie z.B. die am Wank, die einen mittleren Jahresniederschlag von etwa $1500 \mathrm{~mm}$ ausweist, ermöglichen zudem die klare Präferenz für das Morrison-Schema, da dieses mit einem NS von $1290 \mathrm{~mm}$ in diesem Bereich, gegenüber $2300 \mathrm{~mm}$ im Thompson-Schema, die eindeutig belastbareren Werte liefert. Die Diskrepanz der Ergebnisfelder macht deutlich, 


\section{Originalarbeit}

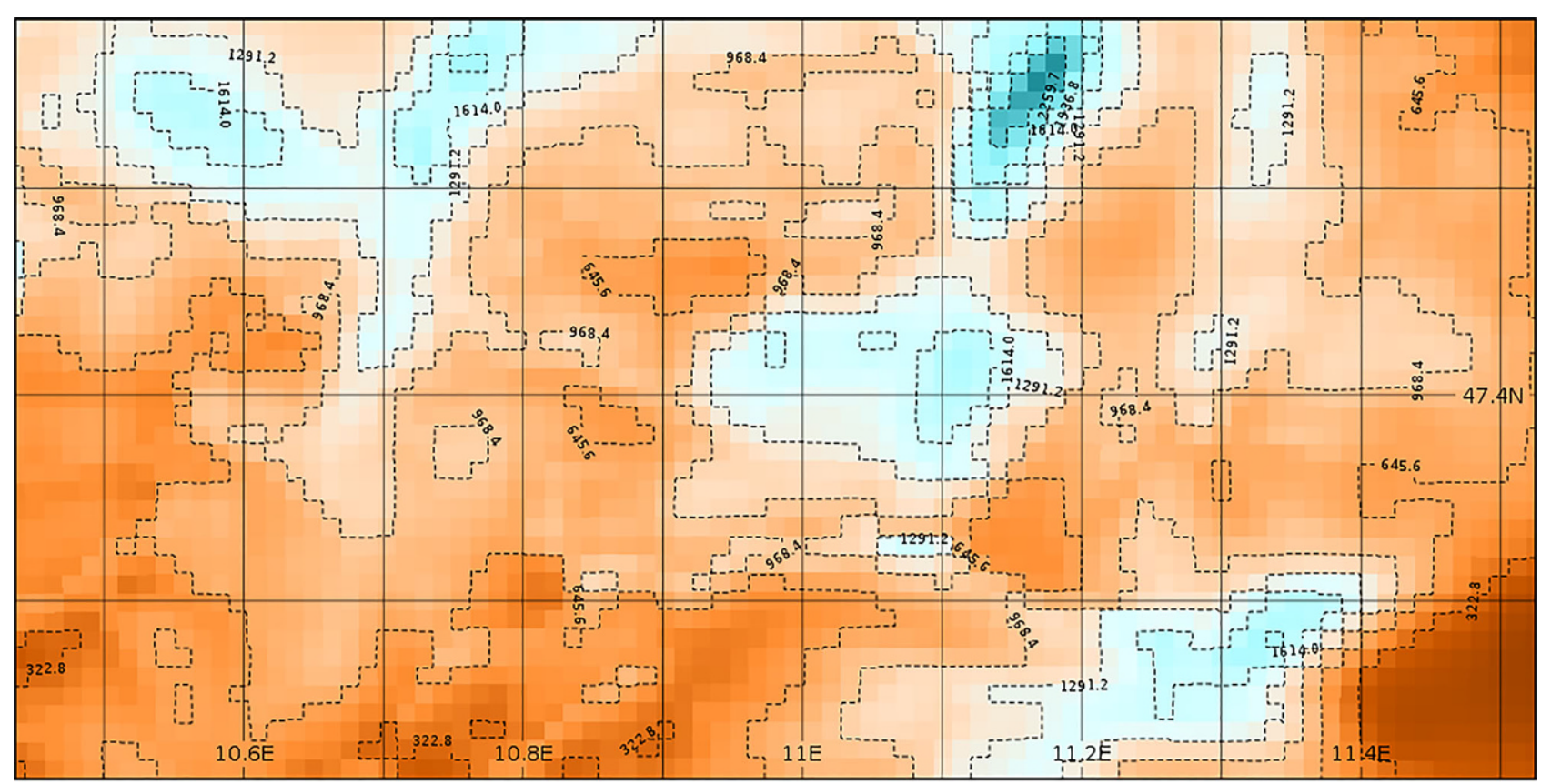

Combined large scale and convective rain, snow and graupel (accumulated) $(\mathrm{kg} \mathrm{m}-2)$

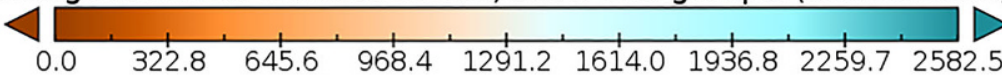

Data $\operatorname{Min}=0.0 . \operatorname{Max}=2582.5$

Abb. 7 Niederschlagsfeld auf Basis des Mikrophysik-Schemas von Thompson et al. (2008)

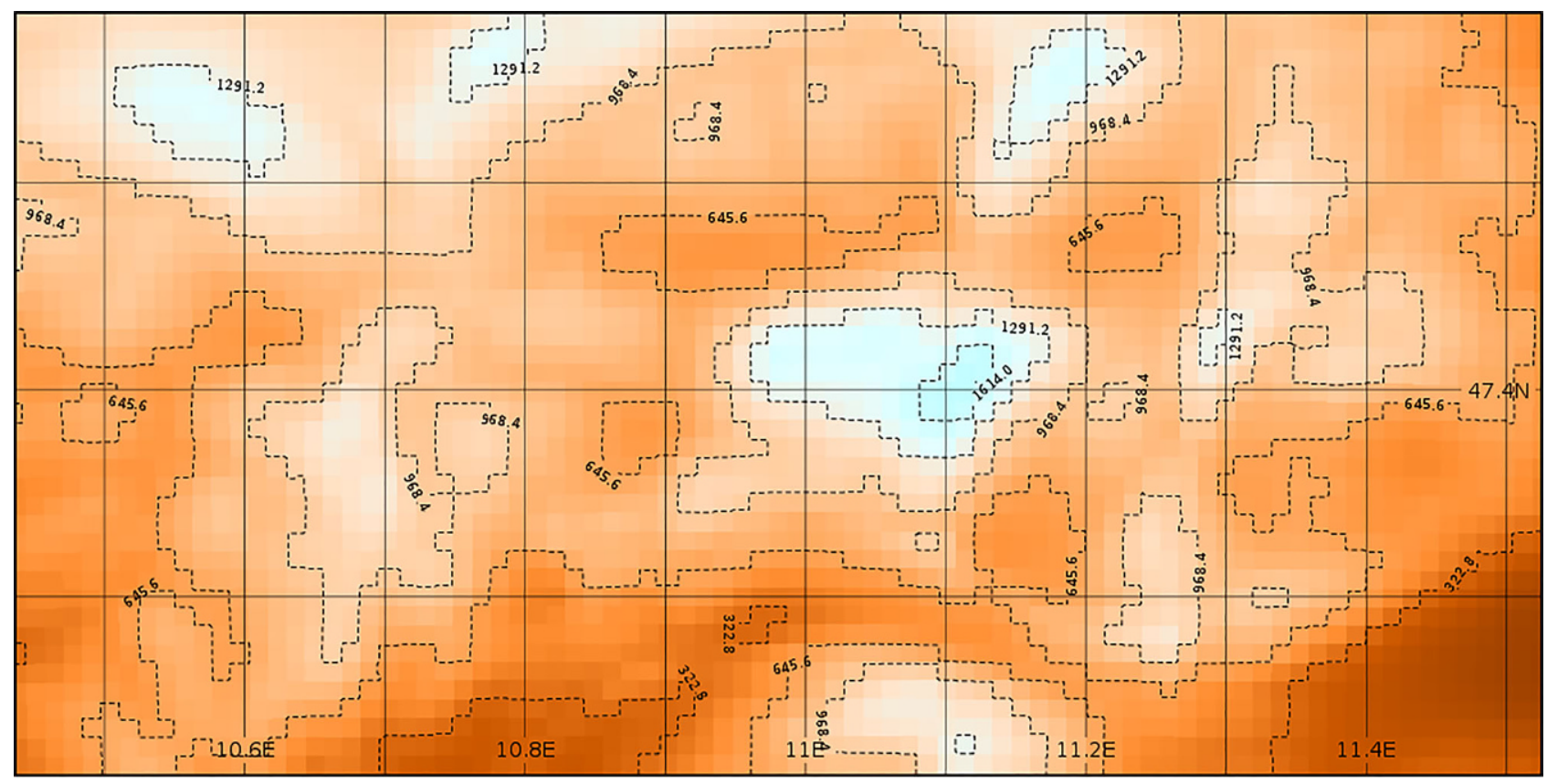

Combined large scale and convective rain, snow and graupel (accumulated) $(\mathrm{kg} \mathrm{m}-2)$

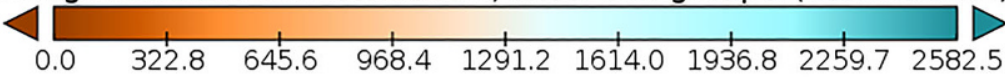

Data $\operatorname{Min}=0.0 . \operatorname{Max}=1718.8$

Abb. 8 Niederschlagsfeld auf Basis des Mikrophysik-Schemas von Morrison et al. (2005a) 


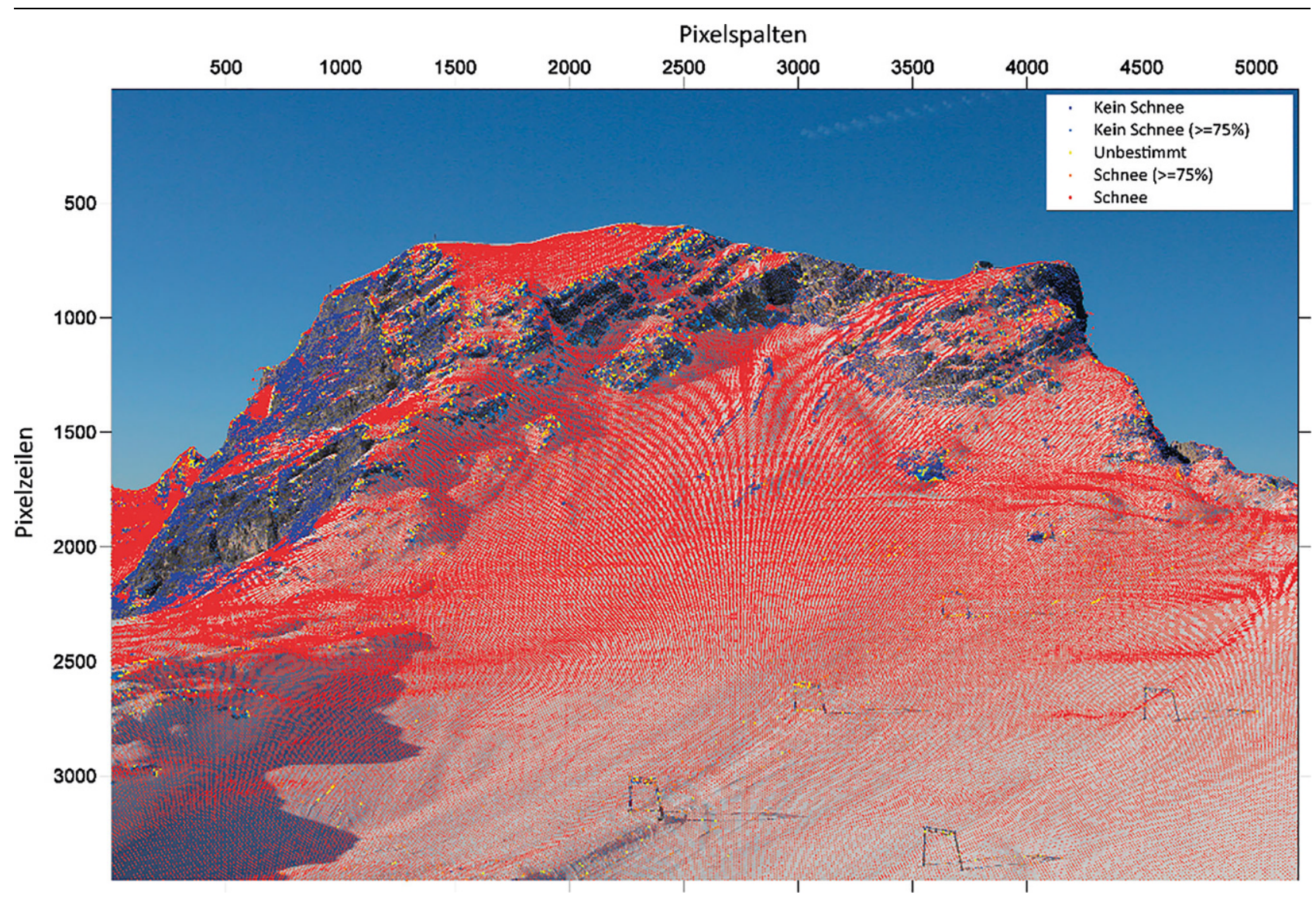

Abb. 9 Projektion des DGM in die Kameraaufnahme vom 17.11.2011 und automatische Klassifikation in schneefreie und schneebedeckte sowie in drei weitere Kategorien für nicht eindeutig bestimmbare Pixel

dass vor der endgültigen Modellierung die adäquaten, auf das jeweilige Gebiet passenden Schemata im Modell selektiert werden müssen. Geschieht dies nicht, wird dies unweigerlich $\mathrm{zu}$ extremen Unsicherheiten in den dann erzeugten Prognosedaten führen.

Um eine solche Evaluation im Gebirgsraum durchführen zu können, sind gerade Höhenstationen essenziell, da die Unterschiede in den Feldern in den Höhenlagen deutlicher zutage treten und eine Evaluierung einzig anhand der Talstationen damit unmöglich erscheint. Die Analyse im Bereich der Parametrisierung der jeweiligen Schemata in Bezug auf die Schneefallgeschwindigkeit und die Cloud Droplet Concentration hat im Weiteren ergeben, dass eine Veränderung dieser Parameter die Ergebnisse noch einmal um jeweils etwa $10 \%$ zu verändern vermögen, sich an der generellen Verteilung der Niederschläge jedoch nichts verändert.

\subsection{Fallbeispiel III}

Die Kalibrierung der NDSI-Schwellenwerte in Satellitenbildern mithilfe von Kameraaufnahmen ermöglicht die Erzeugung hochgenauer Schneebedeckungskarten aus Satellitenbildaufnahmen für den Beobachtungsort und jeden Zeitpunkt, der über eine geeignete Aufnahme adressiert ist. Die Software PRACTISE ermöglicht dabei eine schnelle und unkomplizierte Prozessierung (s. Abschn. 3.3.).

Eine Voraussetzung für die Kalibrierung ist, dass die Projektion und die Schneeklassifizierung der Kameraaufnahme hohen Qualitätsansprüchen genügen. Das Beispiel vom 17.11.2011 in Abb. 9 zeigt die Projektion des Digitalen Geländemodells (DGM) in die Aufnahme einer Spiegelreflexkamera und die dortige Klassifikation der Pixel. Die Ortsgenauigkeit der Projektion ist gerundet $1 \mathrm{~m}$ und somit exakt identisch mit der Auflösung des DGM. Die Genauigkeit der automatischen Klassifikationsergebnisse ist trotz des Schattenwurfs im Bild sehr hoch. Der Fehler liegt bei etwa $0,1 \%$. Ähnliche Genauigkeiten wurden auch für die 15 weiteren untersuchten Aufnahmezeitpunkte gefunden.

Der kalibrierte NDSI-Schwellenwert des Landsat-7-Satellitenbildes vom 17.11.2011, aufgenommen in der gleichen Stunde wie die Kameraaufnahme, liegt bei 0,18. Die Übereinstimmung der Schneekarten aus Kamera und Satellitenbild nach Kalibrierung des NDSISchwellenwertes liegt für das Beispieldatum bei etwa 93,5\%. Dieser Wert entspricht auch dem Mittelwert für die anderen Zeitpunkte. In Anbetracht der unterschiedlichen Auflösung von Kamera $(1 \mathrm{~m})$ und Landsat $(30 \mathrm{~m})$ und dem daraus entstehenden Problem von Mischpixeln, ist dieser Wert als sehr gut anzusehen. Abb. 10 bestätigt diese Aussage auch visuell. Die ausgeprägten Schattenflächen in der Abbildung sind dem Aufnahmezeitpunkt von $10 \mathrm{Uhr}$, der Komplexität des Geländes im Zugspitzgebiet und dem extrem niedrigen Sonnenstand im November geschuldet. In Sommeraufnahmen sind die Schattenflächen deutlich weniger prominent. 


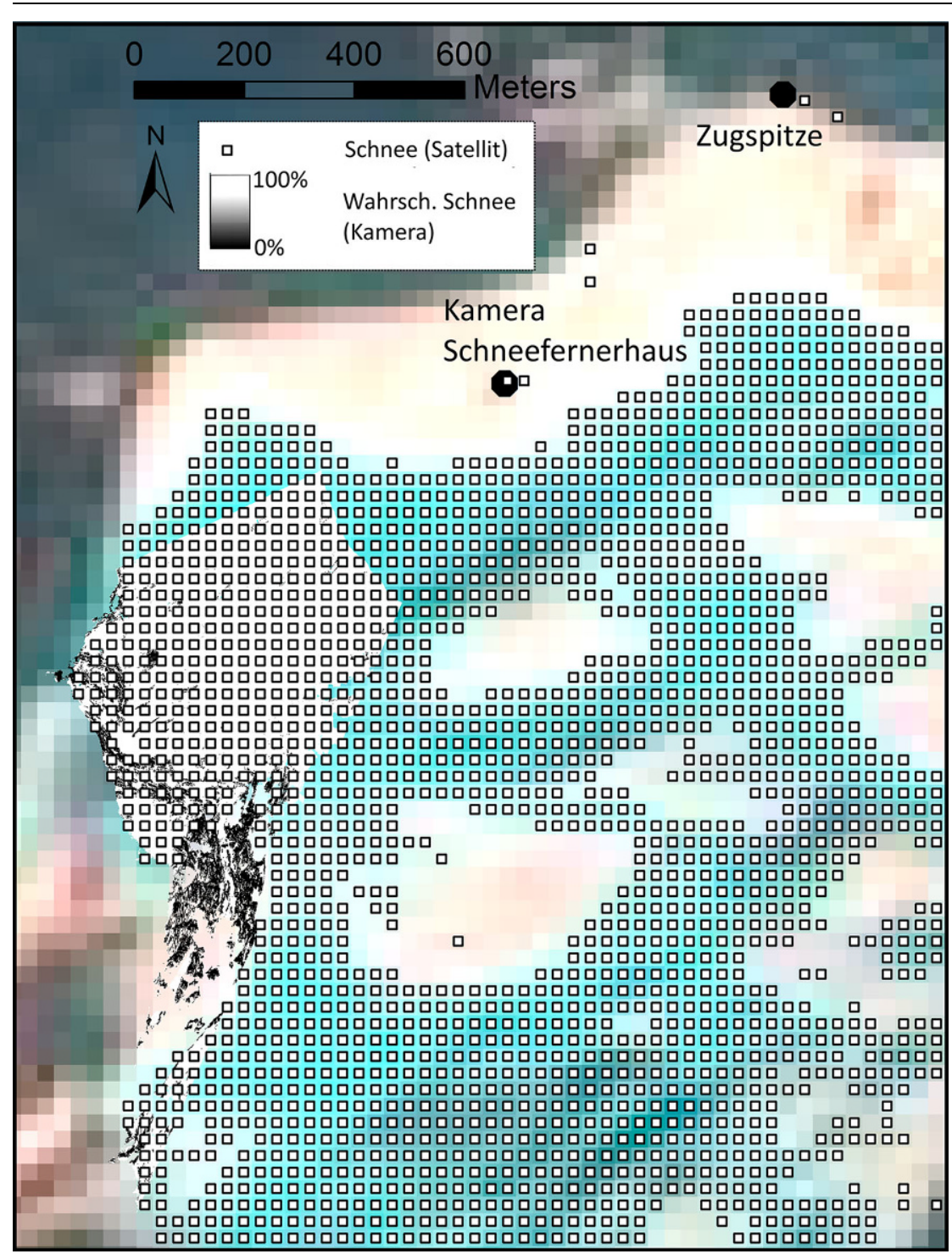

Abb. 10 Überlagerung der kalibrierten Satellitenbildschneebedeckungskarte und der zur Kalibrierung verwendeten Kameraschneekarte für das Zugspitzgebiet am 17. November 2011. Die projizierte Kameraaufnahme zeigt in Rot die Schneebedeckung und in Blau schneefreie Flächen. Die Schneeflächen in der Landsat-7-Aufnahme nach der Kalibrierung mit der Kameraaufnahme sind als weiße Kreuze dargestellt, Gebirgs- und Wolkenschatten sind von der Analyse ausgenommen und werden deshalb maskiert (schwarze Kreuze). Schneefreie Flächen im Satelliten sind somit alle Flächen, in denen das zur besseren Visualisierung verwendete LandsatLook-Bild ohne überlagernde Kreuze zu sehen ist

Für das Beispiel vom 17.11.2011 beträgt der kalibrierte NDSI-Schwellenwert, wie oben bereits erwähnt, 0,18 und ist somit deutlich verschieden zum Literaturwert von 0,4, welcher in den meisten Studien standardmäßig als NDSI-Schwellenwert verwendet wird. Diese Differenz lässt sich auch als Schneeflächendifferenz zwischen beiden Verfahren ausdrücken. Nach der Kalibrierung des NDSI-Schwellenwertes ist die Schneebedeckung im gleichzeitig verfügbaren Kameraaufnahmen und Satellitenbildern betrachtet. Die kalibrierten Schwellenwerte am Zugspitzplatt sind mit 0,28 im Mittel deutlich niedriger als der Standardwert von 0,4. Außerdem ist auffällig, dass für die untersuchten Daten eine große Spannbreite an Werten zwischen 0,15 und $0,39 \mathrm{zu}$ beobachten ist, wobei selbst der maximal auftretende Schwellenwert unter dem Literaturwert liegt. Die Betrachtung der kalibrierten NDSI-Schwellenwerte in Abhängigkeit vom Zeitpunkt im Jahr in Abb. 11 zeigt weiterhin eine hohe Variabilität sowie einen unterliegenden Jahresgang in den Daten.

Die systematisch niedrigeren NDSISchwellenwerte am Zugspitzplatt können mit den Reflektionseigenschaften des Felsuntergrunds, in diesem Fall also Kalkstein, in Zusammenhang gebracht werden. Dies kann auf Basis einer weiteren Studie, die am Vernagtferner in den Ötztaler Alpen durchgeführt wurde (Härer et al. 2018) erklärt werden. Der Untergrund im Vernagtfernergebiet besteht aus Gneiß und die NDSISchwellenwerte für die Abgrenzung von Schnee sind in diesem Gebiet im Mittel deutlich höher $(0,57)$. Die Differenz der NDSI-Werte für Kalkstein und Gneiß beträgt 0,34 und kann somit die Differenz zwischen den Mittelwerten in beiden Gebieten von 0,29 weitgehend erklären. Ebenfalls erklärbar ist der zu beobachtende unterliegende Jahresgang wie das in Abb. 11 gezeigte Modell $\left(\mathrm{R}^{2}=0,57, \mathrm{RMSE}=0,049\right)$ aufzeigt. Trotz des vermeintlich geringen Atmosphäreneinflusses in alpinen Gebieten und der Nutzung eines Index zur Normalisierung von Strahlungsunterschieden, findet sich eine klare Korrelation zwischen den kalibrierten NDSI-Schwellenwerten und dem Azimuth $(0,75)$ bzw. der Sonnenhöhe $(0,77)$. Einzig die hohe Variabilität der NDSI-Schwellenwerte ist somit aktuell noch nicht geklärt. Es liegt die Vermutung nahe, dass eine Kombination aus verschiedenen Faktoren wie Alter und Körnung des Schnees für diese Unterschiede verantwortlich sind (Härer et al. 2018).

Die für diese Studie herangezogenen Landsat-Aufnahmen sind mit $30 \mathrm{~m}$ relativ hochauflösend und somit zur Betrachtung kleiner lokaler Einzugsgebiete optimal. Eine Frage, die sich dabei stellt, ist der Einfluss der räumlichen Auflösung des Satellitenbildes auf die NDSI-Schwellenwerte bzw. die daraus abgeleiteten Schneeflächen. Eine 


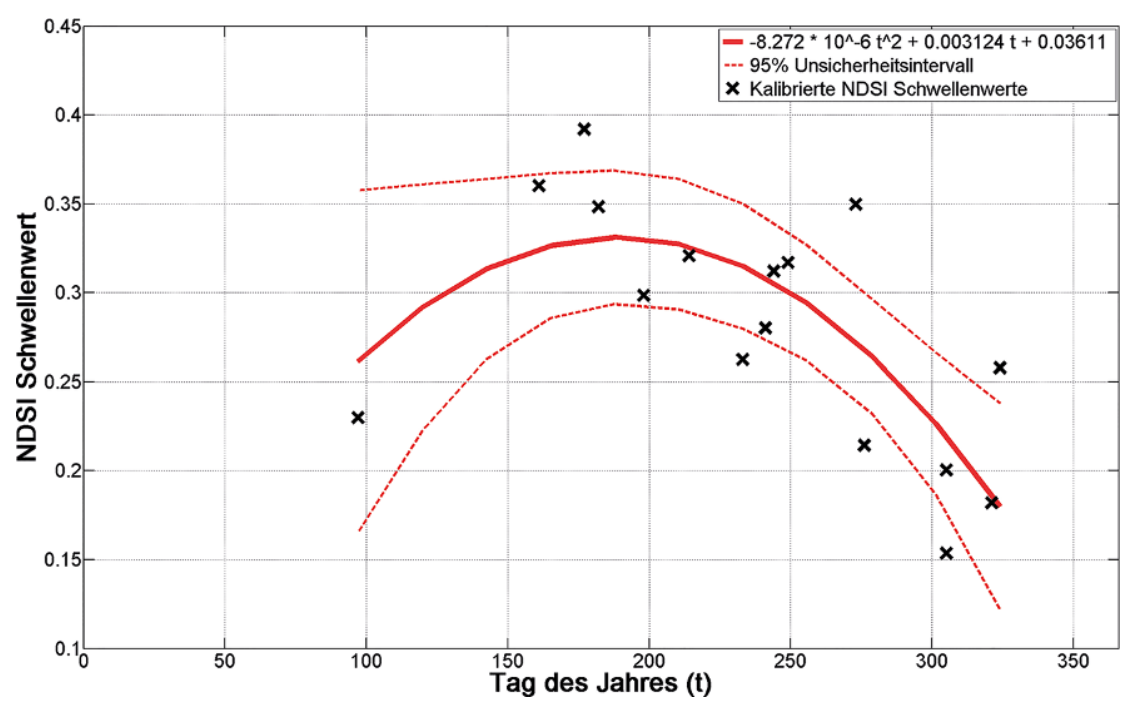

Abb. 11 NDSI-Schwellenwerte (schwarze Kreuze) dargestellt in Abhängigkeit vom Tag im Jahr ( $t$ ). Außerdem sind zur Verdeutlichung des Jahresgangs in den kalibrierten NDSI-Schwellenwerten mit der rot durchgezogenen Linie ein an die Daten angepasstes quadratisches Polynom und mit den rot gestrichelten Linien das 95-\%Unsicherheitsintervall des Modells angetragen

Aggregation der 30-m-Landsat-Daten auf $90,210,510$ und $990 \mathrm{~m}$ zeigt, dass die Unterschiede zwischen den abgeleiteten Schneeflächen aus zeit- und ortsabhängig kalibriertem NDSI-Schwellenwerten und dem Literaturwert von 0,4 mit gröberer räumlicher Auflösung verschwinden. Abb. 12a verbildlicht dies für das Beispiel vom 17.11.2011. Abb. 12b macht den Verlauf der $\mathrm{Zu}$ nahme identischer Schneekarten mit gröberer räumlicher Auflösung und dessen zunehmende Unabhängigkeit vom verwendeten NDSI-Schwellenwert für die gesamte Zeitreihe deutlich. Für 9 bzw. 14 der 16 untersuchten Zeitpunkte finden sich für die Auflösungen von $510 \mathrm{~m}$ bzw. $990 \mathrm{~m}$ keine Unterschiede in den abgeleiteten Schneekarten für das Zugspitzplatt unabhängig vom verwendeten Verfahren.

Es konnte gezeigt werden, dass die NDSI-Schwellenwerte für hochauflösende Satellitenbilder im Zugspitzplatt, einem alpinen Kopfeinzugsgebiet, hochvariabel sind und einen deutlichen Einfluss auf die abgeleiteten Schneeflächen haben. Einige Aspekte der Variabilität der Schwellenwerte (Mittelwert, Jahresgang) sind dabei definitiv systematisch und können beispielsweise dem vorherrschenden Felsuntergrund und dem jeweiligen Sonnenstand zugeordnet werden. Die weitere Variabilität scheint aktuell zufällig, ist aber wahrscheinlich durch andere Faktoren wie Schneealter und Körnung zu erklären, die die Schneealbedo stark beeinflussen. Dies konnte jedoch noch nicht abschließend geklärt werden.

Die Notwendigkeit einer Kalibrierung oder Anpassung des NDSI-Schwellenwerts für den jeweiligen Ort und die jeweilige Zeit ist somit für lokale Schneeflächenuntersuchungen mit hochauflösenden Satellitenbildern eindeutig. Ein weiteres Experiment hat zudem aufgezeigt, dass für Auflösungen von $\geq 500 \mathrm{~m}$ eine Anpassung des Schwellenwertes im Großteil der untersuchten Fälle keine Verbesserung der Schneekarten mit sich bringt. Der zusätzliche Aufwand einer Anpassung des NDSI-Schwellenwerts ist somit in Abhängigkeit zur Skala zu sehen und wird damit nur für lokale und somit hochauflösende Untersuchungen als absolut notwendig betrachtet.

\section{Zusammenfassung}

Die drei genannten Fallbeispiele konnten nur an einem Standort wie dem RCZ durchgeführt werden. Hier ist die not- wendige Infrastruktur installiert und eine ganzjährige Zugänglichkeit gewährleistet. In unseren Augen sind die im RCZ oder ähnlichen Einzugsgebieten erhobenen Daten essenziell. Dies begründet sich in der Tatsache, dass sie als Ankerpunkte alpiner hydrologischer Forschung angesehen werden können. Diese können lokal ein besseres Verständnis darüber ermöglichen, wie sensitiv komplexe Modelle, die eine ausgesprochen gute Instrumentierung voraussetzen, auf verschiedene Geländeparameter reagieren (Fallbeispiel I), wie die Auswahl von Modellkomponenten (Fallbeispiel II) die Güte von regionalen Klimamodellen $\mathrm{zu}$ beeinflussen vermag und wie recht einfache Instrumentierungen ein deutlich verbessertes Fernerkundungsprodukt erzeugen können (Fallbeispiel III). Im Falle der regionalen Klimamodellierung wird darüber hinaus deutlich, dass Messungen aus den Tallagen bei weitem nicht ausreichen, um die Güte der Ergebnisse zu bestimmen, da sich die Unterschiede zwischen den verschiedenen Modellsetups von ICAR v. a. in den Höhenlagen auswirkt. Die Abschätzung der Realitätsnähe des Höhengradienten verlangt aber nach hochqualitativen, hochgelegenen Stationen. Die in Global Cryosphere Watch (GCW) oder INARCH (Pomeroy et al. 2015) organisierten Hochgebirgseinzugsgebiete sowie deren Ausweitung können demnach in Zukunft eine gewichtige Rolle auf dem Weg zu mehr Prozessverständnis und Modellverständnis im Hochgebirge führen.

Funding Open access funding provided by University of Natural Resources and Life Sciences Vienna (BOKU).

Open Access Dieser Artikel wird unter der Creative Commons Namensnennung 4.0 International Lizenz (http:// creativecommons.org/licenses/by/4. $0 /$ deed.de) veröffentlicht, welche die Nutzung, Vervielfältigung, Bearbeitung, Verbreitung und Wiedergabe in jeglichem Medium und Format erlaubt, sofern $\mathrm{Sie}$ den/die ursprünglichen $\mathrm{Au}$ tor(en) und die Quelle ordnungsgemäß nennen, einen Link zur Creative Commons Lizenz beifügen und angeben, ob Änderungen vorgenommen wurden. 

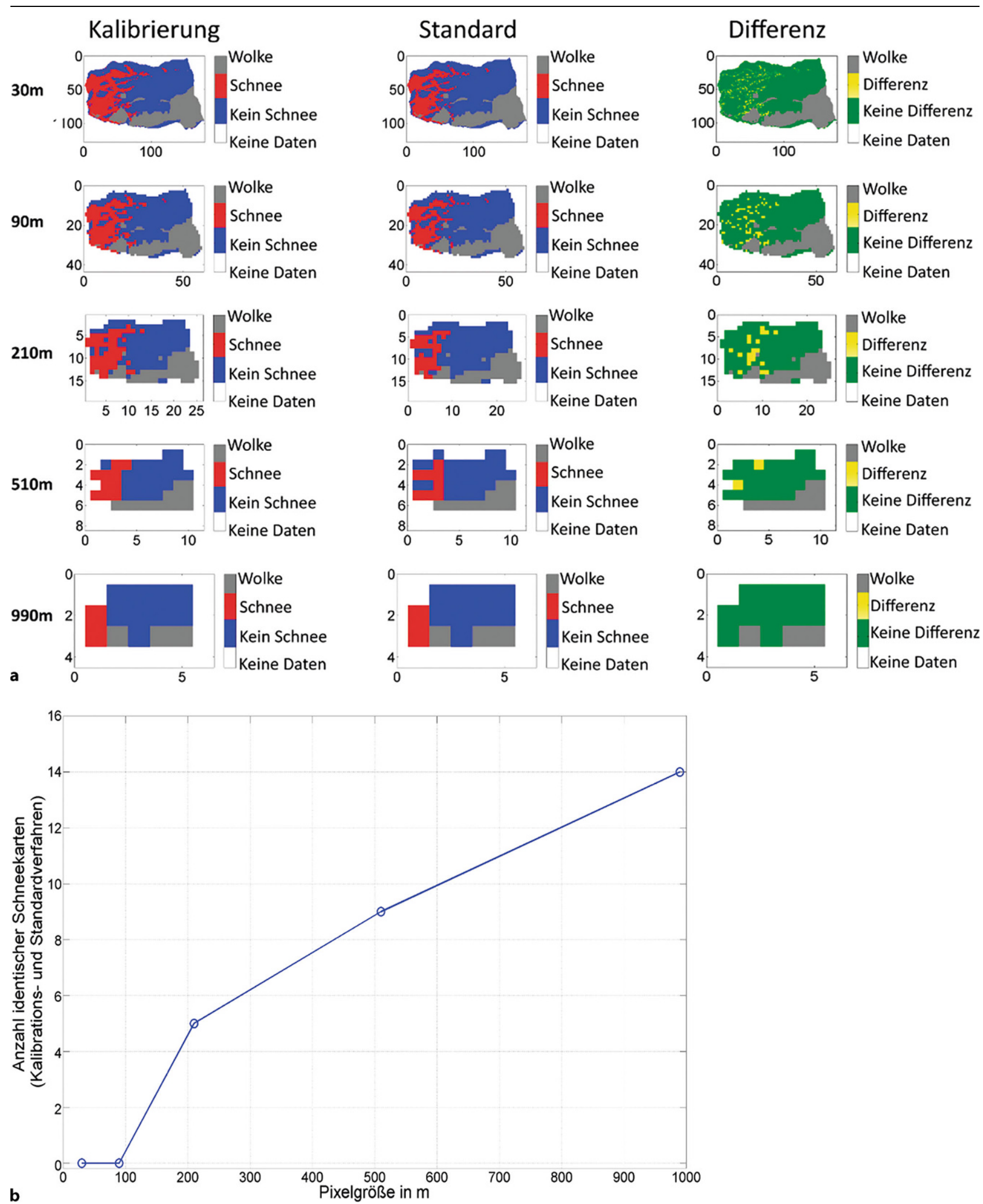

Abb. 12 azeigt den Effekt gröberer räumlicher Auflösung für die aus kalibrierten und aus dem Standard-NDSI-Schwellenwert abgeleiteten Schneekarten am Zugspitzplatt am 17. November 2011 und deren Differenz. In b wird die gesamte Zeitreihe (16 Aufnahmen) betrachtet. Mit der Pixelgröße nimmt die Anzahl identischer Schneekarten zu. Dies ist ein Indiz für die zunehmende Unabhängigkeit der Schneekartenqualität vom verwendeten NDSI-Schwellenwert 
Arsenault, K. R., Houser, P. R., and De Lannoy, G. J. M. (2014): Evaluation of the MODIS snow cover fraction product, Hydrol Process, 28 980-998, doi:10.1002/hyp.9636

Ault, T. W., Czajkowski, K. P., Benko, T., Coss, J., Struble, J., Spongberg, A., Templin, M., and product and cloud mask using student and NWS cooperative station observations in the Lower Great Lakes Region, Remote Sens Environ, 105, 341-353, doi:10.1016/j.rse.2006.07.004

Barstad, I., and Gronas, S. (2006): Dynamical structures for southwesterly airflow over southern Norway: the role of dissipation, Tellus A, $\quad 58$ doi:10.1111/j.1600-0870.2006.00152.x

Bernhardt, M., and Schulz, K. (2010): SnowSlide: A simple routine for calculating gravitational snow transport, Geophys Res Lett, 37, -, Artn L11502, doi: 10.1029/2010gl043086

Bernhardt, M., Harer, S., Jacobeit, J., Wetzel, K. F., and Schulz, K. (2014): The Virtual Alpine Observatory-research focus Alpine hydrology, Hydrol Wasserbewirts, 58, 241-243

Bernhardt, M., Schulz, K., and Pomeroy, J. (2015): The International Network for Alpine Research Catchment Hydrology A new GEWEX crosscutting Project, Hydrol Wasserbewirts, 59, 190-191

Bernhardt, M. (2008): Using wind fields from a high-resolution atmospheric model for simulating snow dynamics in mountainous terrain, $\mathrm{PhD}$, Faculty of Geosciences, Ludwig-Maximilians-Universität (LMU), Munich, 151 pp

Bitner, D., Carroll, T., Cline, D., and Romanov, P. (2002): An assessment of the differences between three satellite snow cover mapping techniques, Hydrol Process, 16, 3723-3733, doi:10.1002/hyp.1231

Dee, D. P., Uppala, S. M., Simmons, A. J., Berrisford, P., Poli, P., Kobayashi, S., Andrae, U., Balmaseda, M. A., Balsamo, G., Bauer, P., Bechtold P., Beljaars, A. C. M., van de Berg, L., Bidlot, J., Bormann, N., Delsol, C., Dragani, R., Fuentes, M., Geer, A. J., Haimberger, L., Healy, S. B. Hersbach, H., Holm, E. V., Isaksen, L., Kallberg, P., Kohler, M., Matricardi, M., McNally, A. P., Monge-Sanz, B. M., Morcrette, J. J., Park, B. K. Peubey, C., de Rosnay, P., Tavolato, C., Thepaut, J. N., and Vitart, F. (2011): The ERA-Interim reanalysis: configuration and performance of the data assimilation system, Q J Roy Meteor Soc 137, 553-597, doi: 10.1002/Qj.828

Dozier, J. (1989): Spectral Signature of Alpine Snow Cover from the Landsat Thematic Mapper Remote Sens Environ, 28, 9-\&

Erickson, T. A., Williams, M. W., and Winstral, A. (2005): Persistence of topographic controls on the spatial distribution of snow in rugged mountain terrain, Colorado, United States, Water Resour Res, 41, W04014, doi:10.1029/2003wr002973 Flügel, W. A. (1995): Delineating Hydrological Response Units by Geographical InformationSystem Analyses for Regional Hydrological Modeling Using Prms/Mms in the Drainage-Basin of the River Brol, Germany. Hydrological Processes 9(3-4), 423-436

Gao, L., Bernhardt, M., and Schulz, K. (2012): Elevation correction of ERA-Interim temperature data in complex terrain, Hydrol Earth Syst Sc, 16, 4661-4673, doi:10.5194/hess-16-46612012

Gao, L., Bernhardt, M., Schulz, K., and Chen, X. W. (2017): Elevation correction of ERA-Interim temperature data in the Tibetan Plateau, Int J Climatol, 37, 3540-3552, 10.1002/joc.4935
Garnier, BJ, Ohmura, A. (1970): The evaluation of surface variations in solar radiation income. Solar Energy, 13, 21-34

Givati, A., Lynn, B., Liu, Y. B., and Rimmer, A. (2012): Using the WRF Model in an Operationa Streamflow Forecast System for the Jordan River J Appl Meteorol Clim, 51, 285-299, doi:10.1175/ Jamc-D-11-082.1

Granger, R.J., Gray, D.M. (1990): A net radiation model for calculating daily snowmelt in open environments. Nordic Hydrology, 21, 217-234

Gray, D.M., Landine, P.G. (1987): Albedo model for shallow prairie snowcovers. Canadian Journal of Earth Sciences 24, 9, 1760-1768

Gutmann, E., Barstad, I., Clark, M., Arnold, J., and Rasmussen, R. (2016): The Intermediate Complexity Atmospheric Research Mode (ICAR), J Hydrometeorol, 17, 957-973, doi:10.1175/Jhm-D-15-0155.1

Gutmann, E. D., Rasmussen, R. M., Liu, C. H., Ikeda, K., Gochis, D. J., Clark, M. P., Dudhia, J., and Thompson, G. (2012): A Comparison of Statistical and Dynamical Downscaling of Winter Precipitation over Complex Terrain, J Climate, 25, 262-281, doi:10.1175/2011jcli4109.1

Harer, S., Bernhardt, M., Corripio, J. G., and Schulz, K. (2013): PRACTISE-Photo Rectification And ClassificaTIon SoftwarE (V.1.0), Geosci Model Dev, 6, 837-848, doi:10.5194/gmd-6-8372013

Härer, S., Bernhardt, M., and Schulz, K. (2016) PRACTISE-Photo Rectification And ClassificaTIon SoftwarE (V.2.1), Geosci. Model Dev., 9, 307-321, doi:10.5194/gmd-9-307-2016

Härer, S., Bernhardt, M., Siebers, M., and Schulz, K. (2018): On the need for a time- and location-dependent estimation of the NDSI threshold value for reducing existing uncertainties in snow cover maps at different scales, The Cryosphere, 12, 1629-1642, https://doi.org/10. 5194/tc-12-1629-2018

Hock, R. (2003): Temperature index melt modelling in mountain areas, J Hydrol, 282, 104-115, doi:10.1016/S0022-1694(03)00257-9

Klemes, V. (1990): The modelling of mountain hydrology: the ultimate challenge, IAHS-AISH P. 190, 29-43.

Kling, H., Fuchs, M., and Paulin, M. (2012): Runoff conditions in the upper Danube basin under an ensemble of climate change scenarios. J Hydrol 424-425, 264-277, https://doi.org/10. 1016/j.jhydrol.2012.01.011

Kreienkamp, F., Spekat, A., and Enke, W. (2013): The Weather Generator Used in the Empirical Statistical Downscaling Method, WETTREG, Atmosphere-Basel, 4, 169-197, doi:10.3390/ Atmos4020169

Lin, Y., Farley, R.D., and Orville, H.D. (1983) Bulk parameterization of the snow field in a cloud model. J Appl Meteor, 22, 1068-1092

Lopez-Moreno, J. I., Boike, J., Sanchez-Lorenzo, A., and Pomeroy, J. W. (2016): Impact of climate warming on snow processes in $\mathrm{Ny}-$ Alesund, a polar maritime site at Svalbard, Global Planet Change, 146, 10-21, doi:10.1016/ j.gloplacha.2016.09.006

Mankin, J. S., Viviroli, D., Singh, D., Hoekstra, A. Y., and Diffenbaugh, N. S. (2015): The potential for snow to supply human water demand in the present and future, Environ Res Lett, 10, Artn 114016, doi:10.1088/1748-9326/10/11/114016

Marks, D., Domingo, J., Susong, D., Link, T., and

Garen, D. (1999): A spatially distributed energy balance snowmelt model for application in mountain basins, Hydrol Process, 13, 1935-1959 Morrison, H., Curry, J. A., and Khvorostyanov, V. I. (2005a): A new double-moment microphysics parameterization for application in cloud and climate models. Part I: Description, J Atmos Sci, 62, 1665-1677, doi: 10.1175/Jas3446.1

Morrison, H., Curry, J. A., Shupe, M. D., and Zuidema, P. (2005b): A new double-moment microphysics parameterization for application in cloud and climate models. Part II: Single-column modeling of arctic clouds, J Atmos Sci, 62, 1678-1693, doi:10.1175/Jas3447.1

Pomeroy, J.W., Dion, K. (1996): Winter radiation exctinction and reflection in a boreal pine canopy: measurements and modelling. Hydrological Processes, 10, 1591-1608

Pomeroy, J., Bernhardt, M., and Marks, D. (2015): Research network to track alpine water, Nature, 521, 32-32

Pomeroy, J. W., Gray, D. M., Brown, T., Hedstrom, N. R., Quinton, W. L., Granger, R. J. and Carey, S. K. (2007): The cold regions hydrological process representation and model: a platform for basing model structure on physical evidence, Hydrol Process, 21, 2650-2667, doi:10.1002/Hyp.6787

Rappl, A., Wetzel, K. F., Buttner, G., and Scholz, M. (2010): Dye tracer investigations at the Partnach Spring (German Alps), Hydrologie und Wasserbewirtschftung, 4, 220-230

Salvatori, R., Plini, P., Giusto, M., Valt, M., Salzano, R., Montagnoli, M., Cagnati, A., Crepaz, G., and Sigismondi, D. (2011): Snow cover monitoring with images from digital camera systems, Ital J Remote Sens, 43, 137-145, https://doi.org/ $10.5721 /$ ItJRS201143211

Sicart, J.E., Pomeroy, J.W., Essery, R.L.H., Hardy, J.E., Link, T., and Marks, D. (2004): A sensitivity study of daytime net radiation during snowmelt to forest canopy and atmospheric conditions. Journal of Hydrometeorology, 5, 744-784 Smiatek, G., Kunstmann, H., Knoche, R., and Marx, A. (2009): Precipitation and temperature statistics in high-resolution regional climate models: Evaluation for the European Alps. J Geophys Res, 114, D19, https://doi.org/10.1029/ 2008JD011353

Smith, R. B. (1979): The influence of mountains on the atmosphere, Advances in Geophysics, 21 doi:10.1016/S0065-2687(08)60262-9

Smith, R.B., Barstad, I. (2004): A linear theory of orographic precipitation, Journal of the Atmospheric Sciences, 61, 12, 1377-1391

Spekat, A., Enke, W., and Kreienkamp, F. (2007) Neuentwicklung von regional hoch aufgelösten Wetterlagen für Deutschland und Bereitstellung regionaler Klimaszenarios auf der Basis von globalen Klimasimulationen mit dem Regionalisierungsmodell WETTREG auf der Basis von globalen Klimasimulationen mit ECHAM5/MPIOM T63L31 2010 bis 2100 für die SRESSzenarios B1, A1B und A2. Publikationen des Umweltbundesamtes

Thompson, G., Field, P. R., Rasmussen, R. M. and Hall, W. D. (2008): Explicit Forecasts of Winter Precipitation Using an Improved Bulk Microphysics Scheme. Part II: Implementation of a New Snow Parameterization, Mon Weather Rev, 136, 5095-5115, doi:10.1175/2008mwr2387. Tolson, B. A., and Shoemaker, C. A. (2007): Dynamically dimensioned search algorithm for computationally efficient watershed model calibration, Water Resour Res, 43, Artn W01413, doi:10.1029/2005wr004723

Viviroli, D., Durr, H. H., Messerli, B., Meybeck, M., and Weingartner, R. (2007): Mountains of the world, water towers for humanity: Typology, mapping, and global significance, Water Resour Res, 43, Artn W07447, doi:10.1029/2006wr005653 Viviroli, D., Archer, D. R., Buytaert, W., Fowler,

H. J., Greenwood, G. B., Hamlet, A. F., Huang, Y., Koboltschnig, G., Litaor, M. I., Lopez-More- 


\section{Originalarbeit}

no, J. I., Lorentz, S., Schadler, B., Schreier, H., Schwaiger, K., Vuille, M., and Woods, R. (2011): Climate change and mountain water resources: overview and recommendations for research, management and policy, Hydrol Earth Syst Sc, 15, 471-504, doi:10.5194/hess-15-471-2011

Wada, Y., van Beek, L. P. H., Viviroli, D., Durr, H. H., Weingartner, R., and Bierkens, M. F. P. (2011): Global monthly water stress: 2 . Water demand and severity of water stress, Water Resou Res, 47, Artn W07518, doi:10.1029/2010wr009792 Wang, J., Robinson, G. J., and White, K. (2000): Generating viewsheds without using sightlines, Photogramm Eng Remote S, 66, 87-90
Warrach-Sagi, K., Schwitalla, T., Wulfmeyer, V. and Bauer, H. S. (2013): Evaluation of a climate simulation in Europe based on the WRF-NOAH model system: precipitation in Germany, Clim Dynam, 41, 755-774, doi:10.1007/s00382-0131727-7

Watt, A., and Watt, M. (1992): Advanced Animation and Rendering Techniques: Theory and Practice, ACM Press, New York, NY, USA, 472 pp. Weber, M., Bernhardt, M., Pomeroy, J. W., Fang, X., Harer, S., and Schulz, K. (2016): Description of current and future snow processes in a small basin in the Bavarian Alps, Environ Earth Sci, 75, ARTN 1223, doi:10.1007/s12665-016-6027-1
Zehe, E., Ehret, U., Pfister, L., Blume, T., Schroder, B., Westhoff, M., Jackisch, C., Schymanski, S. J., Weiler, M., Schulz, K., Allroggen, N., Tronicke, J., van Schaik, L., Dietrich, P., Scherer, U., Eccard, J., Wulfmeyer, V., and Kleidon, A. (2014): HESS Opinions: From response units to functional units: a thermodynamic reinterpretation of the HRU concept to link spatial organization and functioning of intermediate scale catchments, Hydrol Earth Syst Sc, 18, 4635-4655, doi:10.5194/hess-18-4635-2014 